\title{
Novel gold nanoparticle-protein-semiconductor quantum dot hybrid system: synthesis, characterization and application in cancer cell imaging
}

\author{
Runjun Sarma $^{1, *}$, Monoj KumarDas ${ }^{2}$, Lakshi Saikia ${ }^{3}$, Ratul Saikia ${ }^{4}$ \\ ${ }^{1}$ Department of Physics, Mehr Chand Mahajan DAV College for Women, Sector-36, Chandigarh, India \\ ${ }^{2}$ Cancer Genetics and Chemoprevention Research Group, Department of Molecular Biology and Biotechnology, \\ Tezpur University, Assam-784028, India \\ ${ }^{3}$ Advanced Materials Group, Material Science and Technology Division, CSIR-North East Institute of Science and \\ Technology, Jorhat, Assam-785006, India \\ ${ }^{4}$ Biological Science and Technology Division, CSIR-North East Institute of Science and Technology, Jorhat, \\ Assam-785006, India \\ *runjun2018chd@gmail.com
}

DOI 10.17586/2220-8054-2021-12-6-749-762

\begin{abstract}
Formation of a hybrid system by metallic nanoparticle, protein (or peptide), and a semiconductor QD can be a new and alternate material which may be used for biological applications including cancer cell detection and treatment. Herein, we report on the colloidal synthesis of metal-protein-QD hybrid system considering Au NPs, CdSe QDs, BSA and Lysozyme protein. We demonstrate the structural, optical and vibrational properties of Au-CdSe, Au-BSA-CdSe and Au-Lysozyme-CdSe hybrid systems following their use as cancer cell markers. The study of photoluminescence spectra reveals the predominance fluorescence resonance energy transfer (FRET) between CdSe QDs and Au NPs, Au-BSA and Au-Lysozyme complex. The energy transfer efficiency between QDs donor and the Au NPs, Au-BSA, Au-Lysozyme acceptors are estimated to be 46\%, $94 \%$ and 64\%; respectively. Fluorescence imaging results represent high biocompatibility and fluorescent behavior of the QDs and its hybrid system in the MDA-MB-231 breast cancer cells. The calculation of corrected total counts of fluorescence (CTCF) predicts the higher uptake of CdSe QDs as compared to the Au-QDs, Au-BSA-QDs, Au-Lysozyme-QDs by the cells. The significantly varied zeta potential values of the hybrid systems influence the cellular uptake processes. The fabrication of biocompatible (water soluble, biologically stable, having bioconjugation capability, low cytotoxic to the normal cells, fluorescent in biological environment) Au-CdSe, Au-protein-CdSe hybrid systems would open up an alternative strategy in nanobiotechnology, due to their special physical, optical as well as chemical properties.
\end{abstract}

Keywords: Semiconductor QDs, Metallic Nanoparticles, Nanoparticle-QDs hybrid system, Protein, Cancer Cell imaging.

Received: 22 September 2021

Revised: 9 November 2021

\section{Introduction}

Cancer is becoming one of the leading causes of death and the number of cancer cases worldwide is expected to reach 22 million in the next two decades [1]. Although different treatment options are available, including, surgery, chemotherapy, radiation etc., they cannot be considered as completely effective because of lack of high sensitivity and toxicity response to the non-cancerous normal tissue [2]. However, these treatments result in slow progression of the disease and hence increasing the duration for the survival of the patient. In this regard, the use of nanotechnology-based cancer therapies have received considerable attention in recent years because of: early detection with high sensitivity and efficacy, target specific drug delivery, high in vivo imaging resolution etc. [3,4]. In recent years, metallic nanoparticles in general, and gold nanoparticles (Au-NPs) in particular have attracted considerable interest in nanotherapeutic cancer cell treatment as targeting ligands, contrast agents for cancer imaging, therapeutic drugs etc. [5-8].This is due to their unique optical properties, chemical stability, easy synthesis and functionalization, potential biocompatibility, size and surface-dependent properties etc. $[9,10]$. Moreover, Au NPs do not show cytotoxic effect or severe damage on normal cells even when administered orally for a specific period of time [11].

Semiconductor nanocrystals, or quantum dots (QDs) on the other hand, have been considered as efficient luminescent probes and labels for numerous biological applications including target specific cancer therapy owing to their special optoelectronic properties, such as, size-tunable light emission, high signal brightness, long term photostability and narrow emission line width [12,13]. Amongst quantum dots (QD), cadmium selenide (CdSe), is remarkable, because of its various advantages, including band gap tunability and bright emission behavior [14]. Again, the cytotoxicity of ionic cadmium $\left(\mathrm{Cd}^{2+}\right)$ in nanosized CdSe and CdTe has been reported to be appreciably less as compared to elemental cadmium [15]. In addition, cytotoxicity in the cellular studies is mainly contributed from the surface 
modifier rather than CdSe or CdTe core [16]. Consequently, there exist plentiful scope for safe use of nanoscale CdSe in biological systems [17-19] without causing severe damage to normal cells.

Metallic NPs-semiconductor QDs, on the other hand, formed as a result of interaction of quantum confined electrons (excitons) in the QDs and dielectric confined electromagnetic modes (localized surface plasmon resonance (LSPR)) of metallic NPs, opens up their utilization in various novel technological applications in recent years [20]. This is due to their extraordinary optical properties which includes plasmon-induced fluorescence enhancement and quenching [21], plasmon-assisted Forster energy transfer [22], induced exciton-plasmon photon conversion [23] generation of a single plasmons and so on. The study of energy transfer mechanism in metal-QDs systems has great potential in the areas of luminescence tagging, imaging, medical diagnostics, multiplexing, biosensors etc. Until recently, according to various reports, among various semiconductor QDs, CdSe-QDs were proven to act as an effective donor in a fluorescence resonance energy transfer (FRET) mechanism [24].

Biocompatibility is the property of a material that describes its compatibility with the biological particles such as, protein, cells tissue etc. The prerequisites of a NP or a QD to be biocompatible are water solubility, biological environment stability (low aggregation), bioconjugation capability (biological affinity), low cytotoxicity for the normal cells, etc. Biocompatible water soluble, stable NPs/QDs can be achieved through surface modification and functionalization. The different surface modification processes to make biocompatible NPs/QDs are: i) Use of different polymer [25,26], surfactant [27] ii) Capping of the NPs by suitable ligand molecule [28,29] etc. iii) Surface silanization [30] etc. The high adsorption capacity of nanoparticle in biological environment signifies an increased bioconjugation capability and thus biocompatibility of the nanoparticles. Nanoparticles (NPs) have significant adsorption capacities due to their relatively larger surface area. Thus, they are able to bind or carry other molecules such as drugs, probes and proteins attached to their surface by covalent bonds or by adsorption. By attaching specific chemical compounds, peptides or proteins to the surface, the physicochemical properties including charge, hydrophobicity etc. of the NPs can be altered. With the exposure of Au NPs to biological fluid environments, proteins are easily adsorbed onto the surface. This results in a significant variation of the surface property of the Au NPs as well as physiological functions of the adsorbed proteins [31-33]. Similarly, conjugation of QDs, such as CdSe with different biological molecules, including proteins can create a system with desirable luminescent and bio-compatible properties [34,35]. A protein-conjugated NP or QD, results in various special properties of the protein - NP (or QD) system which is based on the characteristics of the conjugated proteins [36,37]. Bovine Serum Albumin (BSA) is the most abundant protein (molecular mass $\sim 66.5 \mathrm{kDa}$, consisting of $\sim 583$ amino acid) in cow-based plasma. It is extensively used in various bio-nanotechnology applications including sensing, imaging etc [38]. Moreover, BSA-conjugated NPs are more stable against flocculation, show better quantum yield and low toxicity. Similarly, Lysozyme proteins (molecular mass $\sim 14 \mathrm{kDa}$, consisting of $\sim 129$ amino acids) that are found in cow/human milk, saliva, serum, tears, hen egg whites have various biomedical applications due to their special antibacterial, anti-inflammatory and anti-cancer properties [39].

In the present work, we describe simple colloidal routes for the synthesis of polyvinyl pyrrolidone (PVP) and sodium dodecyl sulfate (SDS) capped Au NPs and highly fluorescent CdSe QDs; respectively. We report also here the formation of a Au NPs-CdSe QDs hybrid system. The structural, optical, vibrational properties and zeta potential measurement of the $\mathrm{Au}, \mathrm{CdSe}, \mathrm{Au}-\mathrm{CdSe}$ hybrid systems are studied. The influence of protein molecules on the aforementioned properties was investigated by inclusion of BSA and Lysozyme proteins to the Au-CdSe. In addition, we have highlighted the energy transfer behavior of the CdSeQDs to Au NPs, Au-BSA, Au-Lysozyme by considering FRET. Finally, a critical evaluation on the cellular uptake of the CdSe QDs, Au-CdSe s, Au-BSA-CdSe, Au-Lysozyme-CdSe hybrid systems by MDA-MB-231 breast cancer cells lines are highlighted through studies of fluorescence imaging and cytotoxicity.

Forming a biocompatible hybrid system with metallic nanoparticle, protein (or peptide), and a semiconductor quantum dot can be a new and alternate material which may be used for biological applications including cancer cell detection and treatment [39].

\section{Experimental Details}

\subsection{Synthesis of Au Nanoparticles}

Polyvinylpyrrolidone (PVP) is used for the surface modification of the Au NPs to allow their stabilization in the dispersion media. The polymers cause steric repulsion and prevent aggregation of the colloid particles, making them good stabilizer. PVP is widely accepted as a biocompatible polymeric material and it can effectively bind to BSA and Lysozyme proteins [40]. Moreover, PVP can be safely used, as it is nontoxic for normal cells [41].

For the synthesis of PVP surface modified Au NPs, at first, a 1\% PVP solution was prepared by dissolving $0.1 \mathrm{~g}$ of PVP in $10 \mathrm{~mL}$ distilled water with stirring for $30 \mathrm{~min}$. Then, $3.39 \mathrm{~g}$ of gold salt (chloroauric acid, $\mathrm{HAuCl}_{4}$ ) was dissolved in $10 \mathrm{~mL}$ distilled water to prepare $1 \mathrm{mM}$ stock solution. From the stock solution a $0.2 \mathrm{mM}$ solution was 
prepared and added to the PVP solution. After 10 minutes of stirring, freshly prepared $1 \mathrm{~mL}$ of $0.2 \mathrm{mM}$ sodium borohydride $\left(\mathrm{NaBH}_{4}\right)$ in ice cold water was added to the above mixture drop wise. With the addition of $\mathrm{NaBH}_{4}$, the color of the solution spontaneously changes from the light yellow to reddish indicating the formation of the Au NPs by reduction of $\mathrm{HAuCl}_{4}$ by $\mathrm{NaBH}_{4}$.

\subsection{Synthesis of CdSe-SDS QDs}

SDS surfactant-modified CdSe QDs has been found to be less cytotoxic to the normal cells as compared to other surfactants [19]. Here, SDS-capped CdSe QDs (CdSe-SDS) were prepared and washed by reported method [17]. Briefly, a suitable amount of cadmium nitrate-tetrahydrate $\left[\mathrm{Cd}\left(\mathrm{NO}_{3}\right)_{2} \cdot 4 \mathrm{H}_{2} \mathrm{O}\right]$ and SDS were dissolved in deionized Millipore water followed by stirring at $\sim 40^{\circ} \mathrm{C}$ for $20 \mathrm{~min}$. At high $\mathrm{pH}$, the selenium precursor, selenium dioxide $\left(\mathrm{SeO}_{2}\right)$ was reduced by sodium borohydride $\left(\mathrm{NaBH}_{4}\right)$ and mixed with the above solution and stirred for $1 \mathrm{~h}$ at $80^{\circ} \mathrm{C}$ to get CdSe-SDS QDs.

The synthesized PVP-capped Au NPs, SDS-functionalized CdSe QDs, were found to be water soluble and stable in the biological environment. The bio-conjugation capability of the synthesized Au NPs and CdSe QDs were studied by conjugating the nanoparticle/QDs with BSA and Lysozyme proteins as well as by studying their ( $\mathrm{Au}-\mathrm{CdSe}, \mathrm{Au}-$ BSA-CdS, Au-Lysozyme-CdSe systems) fluorescent behavior in the cancer cell environment. Prior to bioconjugation, $\mathrm{Au}$ NPs, CdSe QDs, the colloidal solutions were subjected to centrifugation ( $7000 \mathrm{rpm})$ separately followed by several rinses with deionized water.

\subsection{Synthesis Au-CdSe, Au-BSA-CdSe, Au-Lysozyme-CdSe hybrid system}

For the preparation of Au-CdSe complex, $20 \mathrm{~mL}$ Au NPs solution was mixed with $2 \mathrm{~mL}$ CdSe QDs solution and stirred for $1 \mathrm{~h}$. $5 \mathrm{~mL}$ Au-BSA (or Au-Lysozyme) were prepared by mixing BSA (or Lysozyme) protein (each protein concentration $20 \mu \mathrm{g} / \mathrm{mL}$ )to Au NPs in two separate beakers. This is followed by mild stirring and incubation of the mixtures at $37^{\circ} \mathrm{C}$ for $1 \mathrm{~h}$. The CdSe QDs were conjugated to Au-BSA (or Au-Lysozyme) by stirring for $1 \mathrm{~h}$ at $500 \mathrm{rpm}$ to get Au-BSA-CdSe and Au-Lysozyme-CdSe hybrid system.

The pyrrolidone side group of PVP in Au-PVP NPs may interact with the anionic SDS surfactant coated CdSe QDs. This results the formation of the Au-CdSe hybrid system [42]. Inserting BSA (or Lysozyme) protein in the Au-CdSe complex, there is a possibility of interaction of $\mathrm{H}_{3} \mathrm{~N}^{+}$of group of BSA (or Lysozyme) to the anionic SDS in one side and in the other side COO- part of BSA to the positively charged PVP molecule resulting Au-BSA-CdSe and Au-Lysozyme-CdSe hybrid [43,44]. Fig. 1 shows the schematic figure of possible formation of Au-CdSe (Fig. 1A), Au-BSA-CdSe and Au-Lysozyme-CdSe (Fig. 1B).

\subsection{Cell culture, fluorescent and cytotoxicity study}

Breast cancer cell line MDA-MB-231 was purchased from the National Centre for Cell Science (NCCS), Pune, India. MDA-MB-231 cells were cultured in L-15 medium (Leibovitz) (HiMedia, Mumbai, India) containing $10 \%$ fetal bovine serum, $100 \mathrm{U} / \mathrm{mL}$ penicillin and $100 \mathrm{mg} / \mathrm{mL}$ streptomycin. The cells were washed with phosphate buffer solution (PBS) and harvested with $0.25 \%$ trypsin-EDTA solution and seeded at densities of $2 \times 10^{4}$ cells/well onto cover glasses placed inside a 6-well tissue culture plate. Cells were treated with $25 \mu \mathrm{g} / \mathrm{mL}$ QDs, Au-QD and Au-protein-QD complexes separately for $24 \mathrm{~h}$. After this treatment, the cells were washed twice with $1 \mathrm{X}$ PBS to remove the unattached cells and then $10 \mu \mathrm{L}$ of cell suspension were taken into slides and observed in a fluorescence microscope. The CdSe QDs and its different complexes were used as cancer cell probes by using fluorescent a microscope (Model: LeicaDM300, USA) equipped with a cooled color CCD camera (Model DP71) with $40 \times$ objectives. QD fluorescence was detected using band-pass (BP) excitation and emission filters.

In vitro cytotoxicity of QDs, Au-protein-QD complexes was assessed using a standard MTT (3-(4,5-dimethyl-2thiazolyl)-2,5- diphenyl-2-H-tetrazoliumbromide) assay with cell lines (MDA-MB231 cells) $2 \times 10^{3}$ cells were seeded in a 96-well plate and treated with increasing concentrations 5, 10 and $25 \mu \mathrm{g} / \mathrm{mL}$ of QDs, Au-protein-QD complexes for $24 \mathrm{~h}$ at $37^{\circ} \mathrm{C}, 5 \% \mathrm{CO}_{2}$ Cytotoxicity was determined by adding $10 \mu \mathrm{L}$ of MTT $(0.5 \mathrm{mg} / \mathrm{mL}$ in PBS $)$ to each well and incubated for $4 \mathrm{~h}$. The medium was removed and $200 \mu \mathrm{l}$ DMSO was added to each well and after 10 min of mechanical shaking, the optical density was measured at $570 \mathrm{~nm}$ in plate reader. The viability was determined in relation to control cells cultured in drug-free media. All experiments were repeated at least three times. SEM values were less than $10 \%$.

\subsection{Characterization Techniques}

The structural characterizations of the Au NPs, CdSe QDs and Au-CdSe systems were performed by a highresolution transmission electron microscopy (HRTEM), (Model: JEM-2100) at NEHU working at an accelerating voltage of $200 \mathrm{kV}$. The optical absorption study was performed by the UV-Visible absorption spectroscopy (Model: 

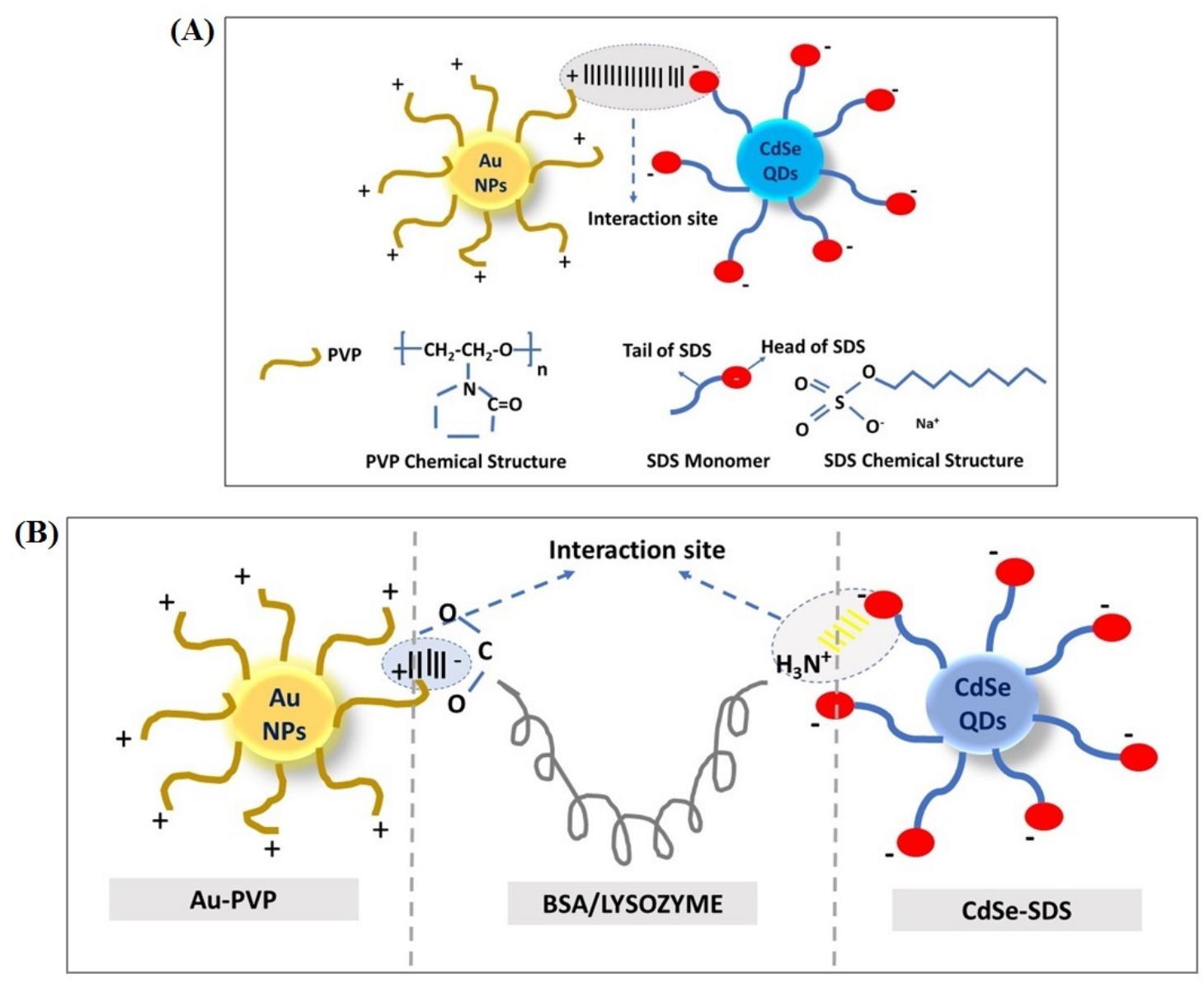

FIG. 1. Schematic diagram of formation of (A) PVA capped Au NP and SDS coated CdSe QD complex (B) Au-BSA-CdSe (or Au-Lysozyme-CdSe) hybrid system

MS-11-UV-1800, Shimadzu Corporation), whereas photoluminescence (PL) spectra were obtained by using a spectrofluorometer (FluoroMax-4CL, HORIBA Scientific). The zeta potential was measured using a Malvern Zetasizer (Model: Nano ZS). Furthermore, IR-active vibrational features were assessed through Fourier transform infrared (FTIR) (Model: NicoletImpact-410) studies. The QDs were used as cancer cell probe by using fluorescent microscope (Model: Leica DM300, USA).

\section{Results and Discussions}

We discuss below the analysis of PVP-capped Au NPs, SDS-coated CdSeQD, Au-CdSe, Au-Protein-CdSe complexes characterized by different techniques.

\subsection{Morphological analysis through transmission electron microscopy studies}

The HRTEM images of the as-synthesized PVP capped Au NPs, CdSe-SDS QDs, and Au-CdSe metallic semiconductor hybrid system are shown in Fig. 2A, 2B and 2C respectively. The Au NPs are found to be fairly hexagonal in shape and each particle are isolated from each other (Fig. 2A(i)). The magnified image of a Au NP is shown in the inset of Fig. 2A(i). Whereas, the obtained CdSe-QDs (Fig. 2B(i)) and Au-CdSe (Fig. 2C(i)) NP-QD systems were nearly spherical in shape. The average size $(D)$ of the Au NPs, CdSe-QDs and Au-CdSe were predicted to be $\sim 2 \mathrm{~nm}$, $\sim 4.68 \mathrm{~nm}$ and $3.8 \mathrm{~nm}$; respectively as obtained from the size distribution histogram (Insets Fig. 2A(i), 2B(i), 2C(i)). The SAED patterns revealed the polycrystalline nature of Au NPs (Fig. 2A,(ii)) [45], CdSe QDs (Fig. 2B,(ii)) [46] and Au-CdSe (Fig. 2C,(ii)) complex. The SAED pattern of Au NPs clearly indicates the growth of lattice planes along (111), (200) and (220) directions with interplanar distance of $\sim 0.23, \sim 0.20, \sim 0.14 \mathrm{~nm}$; respectively. The fast Fourier transform (FFT) of a selected part was taken to measure the lattice plane distances with increased accuracy. Consequently, it signified the lattice plane distance of $0.23 \mathrm{~nm}$ having growth along the (111) direction (upper left inset of Fig. 2A, (ii)). The value of $d_{i}$ of Au NPs resembled the value for the bulk Au system. A magnified, well- resolved 
TABLE 1. Values of Zeta potentials

\begin{tabular}{|l|l|}
\hline Sample & Zeta potential (mV) \\
\hline Au NPs & -13.2 \\
\hline CdSe-SDS QDs & -54 \\
\hline Au NPs-CdSe QDs & -16.9 \\
\hline Au NPs-BSA-CdSe QDs & -16.7 \\
\hline Au NPs-Lysozyme-CdSe QDs & -9.7 \\
\hline
\end{tabular}

lattice planes of a single Au-NP has been shown as in lower right corner of the Figure. In the case of an isolated CdSe-SDS QD, the FFT image (upper left inset of Fig. 2B, (ii))) highlight the distinct diffraction spot corresponding to (101) plane with an inter-planar spacing $\left(d_{i}\right)$ of $\sim 0.35 \mathrm{~nm}$. For the composite Au-QD system, the SAED pattern (Fig.2C(ii)) was found to be different from that of pure Au or pure CdSe QDs system. This indicates that particles are not the pure Au NPs or pure CdSe QDs. The first diffraction ring of the SAED pattern was predicted to be from (111) plane of pure Au NPs, while the second is due to the diffraction from (112) plane of CdSe QDs. We have taken FFT image and produced the intensity plot profile (IPF)[https://imagej.nih.gov/ij/docs/examples/tem/] of the inverse FFT image considering different sections of the TEM image of a single particle to more clear visualization of interplanar spacing in this complex. It is to be noted that the corresponding FFT and IPF of the section A (shown in Fig. 2C,(iii)) clearly indicates the $d_{i}$ of $0.20 \mathrm{~nm}$ of (111) crystallographic orientation of Au NP. Whereas, the section B specifies the presence of CdSe QDs with lattice plane direction along (102) with $d_{i}$ of $0.26 \mathrm{~nm}$. As TEM images are 2D images, the spatial locations of the QDs in the Au NPs are impossible to identify. However, their presence as attached part in the outer side can easily be identified. The increase of average diameter of Au NPs from $\sim 2 \mathrm{~nm}$ to $\sim 3.8 \mathrm{~nm}$ supports this fact. Similar behavior has been observed for $\mathrm{CdS}_{x} \mathrm{Se}_{1-x} / \mathrm{ZnS}$ QDs in Au NPs [47].

\subsection{Measurement of zeta potential}

The magnitude of the zeta potential gives an indication of the potential electrostatic stability of the colloidal system. As the zeta $(\xi)$ potential increases (negative or positive), the repulsion between the constituent particles gets enhanced, resulting in a more stable colloidal dispersion [48]. However, a colloidal solution with nanoparticles having zeta potential values varying in the range of -30 to $\pm 30 \mathrm{mV}$ is generally considered to be stable [49]. In nanodrug delivery systems, the zeta potential not only affects the stability of the colloidal system, but also it dramatically changes their circulation in the blood stream and absorption of the NPs into the cellular membranes [50]. It is to be noted that particles with negative zeta potential values are less cytotoxic as compared to the positive counterparts. This is because of their rapid opsonization and hence clearance by the reticuloendothelial system (RES) in the blood stream [50]. The positively charged NPs, on the other hand, can be cytotoxic due to their cationic charge density and the molecular weight etc.

While studying the zeta potential (Table 1) behavior of the as synthesized NPs, it was observed that all the systems showed negative zeta potential values (Table 1), indicating a good colloidal stability. By conjugating Au NPs with CdSe QDs, the zeta potential values of the Au NPs were improved to $-16.9 \mathrm{mV}$ from $-13.2 \mathrm{mV}$. A zeta potential value of $\sim-15 \mathrm{mV}$ for PVP capped Au NPs has been reported previously [51].

While conjugating BSA protein, the zeta potential of Au-CdSe complex slightly changes to $-16.7 \mathrm{mV}$. On the other hand, significant variation of Zeta potential of Au-CdSe complex occurs to $-9.7 \mathrm{mV}$ from $-16.9 \mathrm{mV}$ while forming the Au-Lysozyme-CdSe complex. The change of the zeta potential values for Au-CdSe system with the inclusion of BSA and Lysozyme protein, signify the efficient conjugation of the respective proteins to the NP-QD complex. Moreover, the reduction of zeta potential value of the parent Au NPs with formation of Au-CdSe, Au-BSACdSe, Au-Lysozyme-CdSe complexes signifies the enhancement of the stability of the Au NPs system.

\subsection{Molecular vibrations in Au NPs, Au-CdSe NPs-QDs, and Au-BSA-CdSe, Au-Lysozyme-CdSe complex}

The FTIR spectra of Au NPs, CdSe-QDs, Au-CdSe, Au-BSA-CdSe, Au-Lysozyme-CdSe complexes are shown in Fig. 3. The common bands observed around $3411 \mathrm{~cm}^{-1}$ and $1630 \mathrm{~cm}^{-1}$ in all the spectra, reveal O-H stretching and an O-H bending vibration of water respectively. Whereas, in the case of PVP-capped Au NPs (Fig. 3, curve, (i)), the sharp band observed at $2122 \mathrm{~cm}^{-1}$ may correspond to the C-H bonding band of Au-PVP NPs which is red shifted from original peak at $2900 \mathrm{~cm}^{-1}$ of pure PVP [52]. The FTIR band due to the $\mathrm{C}=\mathrm{O}$ stretching band of PVP has been observed at $\sim 1650 \mathrm{~cm}^{-1}$. This band is red shifted from original band at $1667 \mathrm{~cm}^{-1}$ contained in the pure PVP. This indicates a charge transfer interaction of PVP molecules with Au surface via O-atom of pyrrolidone ring [53]. Similarly, $\mathrm{CH}_{2}$ twisting $\left(1,230 \mathrm{~cm}^{-1}\right)$ vibrations in the pyrrolidone ring of PVP molecules have been observed at 
(A)

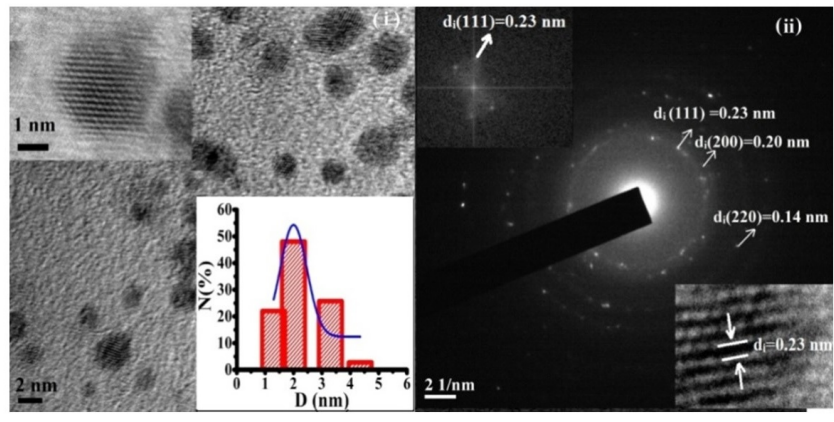

(B)

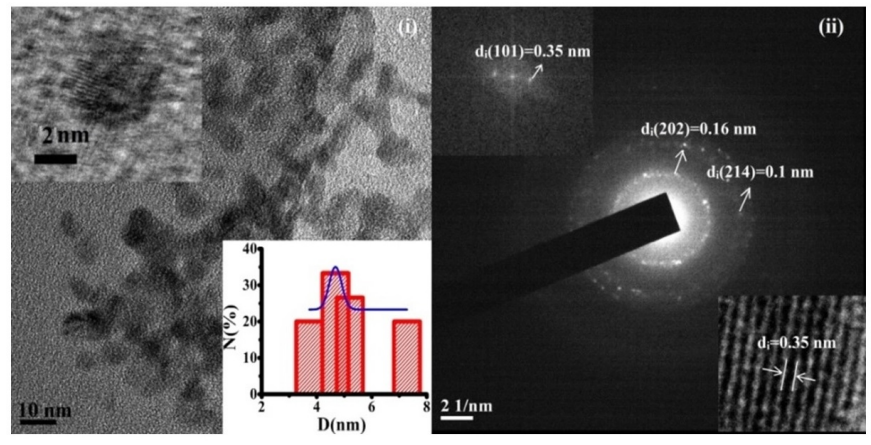

(C)

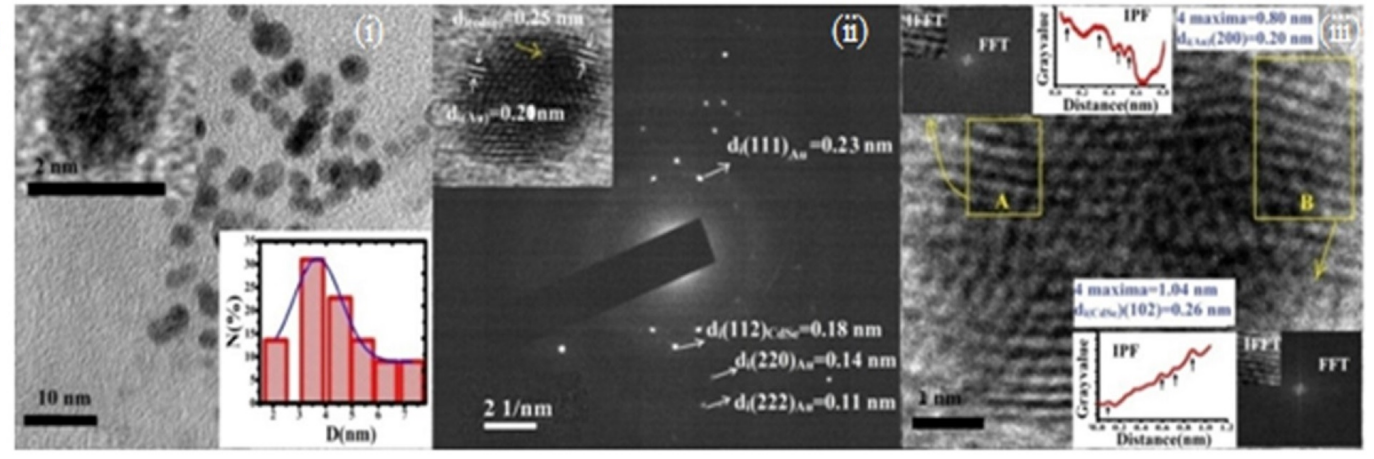

FIG. 2. HR-TEM image of (A) Au NPs (B) CdSe-SDS QDs (C) Au-CdSe NPs-QDs. Particle size distribution histogram are shown in lower right corners and single magnified particle in the upper left corner of the Fig. 1A(i), B(i), C(i). The SAED patterns with of the NPs systems are shown in (ii). The FFT pattern of the Fig. 1A(ii), B(ii) are shown in the upper left corner. Figure 1C(iii) shows the FFT patterns with IPF of different parts of a Au-CdSe complex indicating fromation of $\mathrm{CdSe}$ shell on the outer part of Au NPs

$1192 \mathrm{~cm}^{-1}$ in PVP-capped Au NPs. Fig. 3, curve (ii) shows the FTIR spectra of SDS capped CdSe QDs. It can be observed that, with SDS coating, the peak due to the asymmetric $-\mathrm{CH}_{2}$ stretching $\left(\nu_{\text {asym }}\left(-\mathrm{CH}_{2}\right)\right)$ of the SDS surfactant can be witnessed at $\sim 2919 \mathrm{~cm}^{-1}$. However, the band at $\sim 1524 \mathrm{~cm}^{-1}$ is assigned to the scissoring mode of the SDS surfactant, which is slightly shifted to a higher wave number position wrt $1559 \mathrm{~cm}^{-1}$ of pure SDS molecules. The spectral region located in $1300-1400 \mathrm{~cm}^{-1}$ in SDS is characteristic of the $-\mathrm{CH}_{2}$ wagging modes. This region contains features which are related to gauche conformations. Thus, the band observed at $\sim 1356 \mathrm{~cm}^{-1}$ of the CdSe-SDS QDs can be assigned to the $\mathrm{CH}_{2}$ wagging mode. Whereas, the band corresponding to $\nu_{\text {sym }}\left(-\mathrm{SO}_{3}^{-}\right)$stretching mode of the $\mathrm{SO}_{3}^{2-}$ group is located at $\sim 1207 \mathrm{~cm}^{-1}$. The observed shifting of the $\nu_{\text {sym }}\left(-\mathrm{SO}_{3}^{-}\right)$mode from a value of $1084 \mathrm{~cm}^{-1}$ (pure SDS) to a value of $\sim 1207 \mathrm{~cm}^{-1}$ (for CdSe-SDS QDs), is presumably due to an improved interaction of the headgroups with plentiful $\mathrm{Na}^{+}$counterions. The band observed at $\sim 632 \mathrm{~cm}^{-1}$ is assigned to an effaceable bending of the Cd-Se itself. It can be anticipated that, the enhancement of interaction of the headgroups, as observed in case of SDS-capped QDs may create a stable dispersion of the QDs in the aqueous medium, thereby resulting in the formation of smaller sized QDs with good surface passivation [17,54]. 


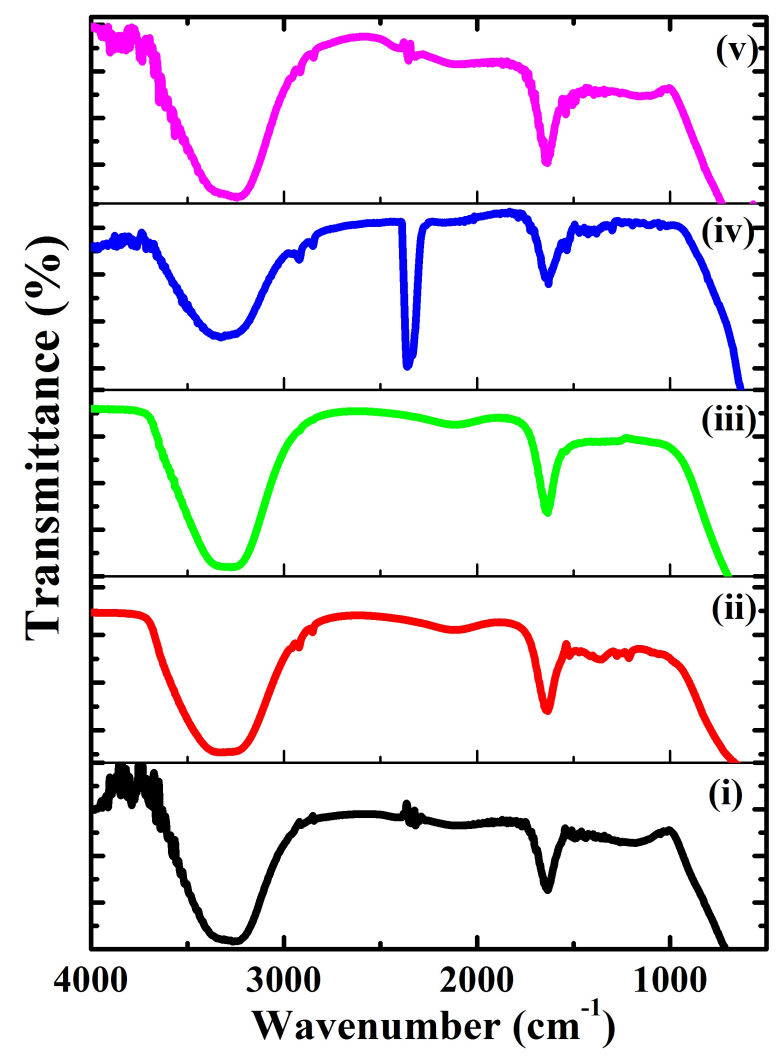

FIG. 3. FTIR spectra of (i) Au NPs (ii) CdSe-QDs (iii) Au-CdSe (iv) Au-BSA-CdSe and (v) AuLysozyme-CdSe hybrid system

The FTIR spectrum of Au-CdSe hybrid system can be depicted from Fig. 3, curve (iii). In this case, the band arises due to C-H bonding of PVP in Au-PVP NPs is slightly blue shifted to $\sim 2360 \mathrm{~cm}^{-1}$ from the original $2122 \mathrm{~cm}^{-1}$ of the parent Au NPs. The band corresponding to the $\nu_{\text {sym }}\left(-\mathrm{SO}_{3}^{-}\right)$stretching mode of the $\mathrm{SO}_{3}^{2-}$ group of SDS molecule was observed at $1268 \mathrm{~cm}^{-1}$ in this case.

Fig. 3,(iv), (v) shows the FTIR spectra Au-BSA-CdSe, Au-Lysozyme-CdSe complex; respectively. In this, the band raised due to $\mathrm{C}-\mathrm{H}$ bonding of PVP can be observed at $2103 \mathrm{~cm}^{-1}$. Whereas, the band at $1654 \mathrm{~cm}^{-1}$ is due to the $\mathrm{C}=\mathrm{O}$ stretching vibration of peptide bond, while the bands at $1535 \mathrm{~cm}^{-1}$ and $1240 \mathrm{~cm}^{-1}$ are due to C-N stretching vibration/N-H bending vibration which are called the amide II band and amide III band, respectively. The band due to the asymmetric $-\mathrm{CH}_{2}$ stretching $\left(\nu_{\text {asym }}\left(-\mathrm{CH}_{2}\right)\right)$ of the SDS surfactant can be witnessed at $\sim 2842 \mathrm{~cm}^{-1}$. While conjugating Lysozyme proteins to Au-CdSe NPs-QDs system, the bond due to amide I and amide II appears with lower intensity along with the appearance of $\mathrm{C}-\mathrm{H}$ bond vibration of PVP and $\mathrm{CH}_{2}$ stretching $\left(\nu_{\text {asym }}\left(-\mathrm{CH}_{2}\right)\right)$ related to SDS. Note that the amide I band has a correlation with the secondary structure of the protein, whereas the amide II is indicative of the amount of protein adsorbed on surfaces. Thus, the arisen of FTIR peaks for amine groups of BSA in addition to the band due to PVP and SDS predicts the conjugation/absorption of BSA and Lysozyme protein to the $\mathrm{Au}-\mathrm{CdSe}$ metal-semiconductor system [55].

\subsection{UV-Vis spectra of Au NPs, Au-CdSe NPs-QDs, and Au-BSA-CdSe, Au-Lysozyme-CdSe hybrid system}

Fig. 4 depicts the UV-Vis absorption spectra of Au NPs, CdSe QDs, Au-CdSe NPs-QDs and different protein conjugated systems. As can be found, Au NPs shows SPR band at $\sim 539 \mathrm{~nm}$ arising from the collective oscillation of free conduction electrons induced by an interacting electromagnetic field. Whereas, a prominent absorption band at $\sim 300 \mathrm{~nm}(4.13 \mathrm{eV})$, has been observed for CdSe-SDS QDs with a blue shifting of $\sim 2.39 \mathrm{eV}$ from the bulk value of $1.74 \mathrm{eV}$. This shows effective quantum confinement of charge carriers in the SDS capped CdSe QDs. In the Au-CdSe hybrid system NPs, the SPR band assigned to Au NPs is observed at $\sim 545 \mathrm{~nm}$ with a red shifting of $\sim 5 \mathrm{~nm}$ from the parent Au NPs system. Moreover, this peak is found to be broadened than the original peak in Au NPs. On the other hand, a slight red shifting to $\sim 303 \mathrm{~nm}$ has been observed for CdSe-QDs peak in the hybrid metal semiconductor NPs system. This broadening of the SPR peak may be due to multipolar excitations and radiative damping in hybrid system [56]. While, the red shifting of plasmonic bands due to the conjugation to QDs suggests a strong interaction 
between the Au NPs and the CdSe QDs [57]. The covering of Au (RI:0.20) NPs core with CdSe (RI:2.5) QDs having larger refractive index (RI) may cause the observed red-shifting of the excitonic feature [58].

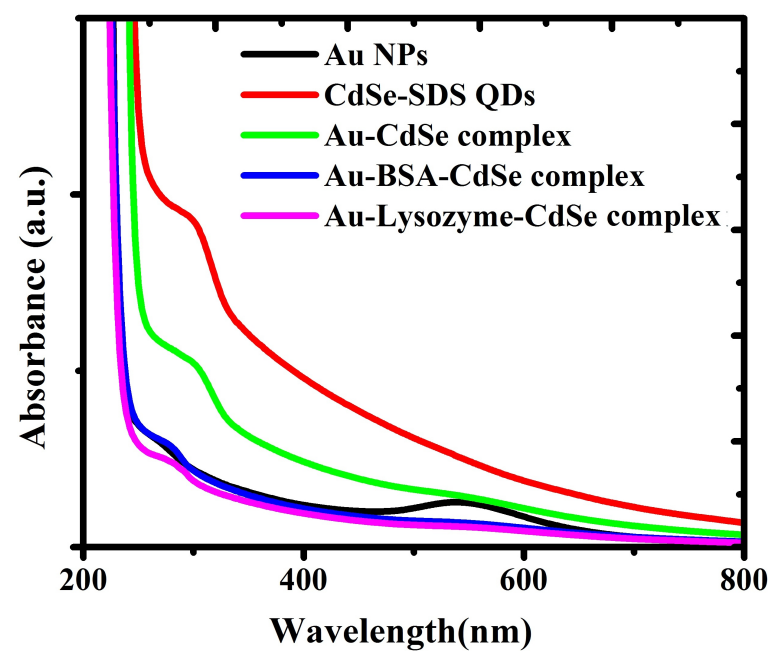

FIG. 4. UV-Vis spectra of Au NPs, CdSe-QDs, Au-CdSe NPs-QDs and their different protein conjugated hybrid systems

Note that, with conjugation of BSA protein, the absorption spectrum shows excitonic features at wavelengths of $277 \mathrm{~nm}$ and $545 \mathrm{~nm}$. The absorption peak at $\sim 277 \mathrm{~nm}$ is attributed to absorption feature of tryptophan molecule of BSA. Similarly, the Lysozyme protein conjugation results in absorption maxima at $\sim 279 \mathrm{~nm}$ and at $\sim 544 \mathrm{~nm}$. The appearance of hump at $\sim 277$ and $279 \mathrm{~nm}$ in case of Au-BSA-CdSe and Au-Lysozyme-CdSe complex can be related to the interaction between serum albumin and AuNPs through a ground state complex formation, caused by the partial adsorption of BSA/Lysozyme proteins on the surface of NPs. Such an adsorption leads to a partial unfolding of the proteins due to the breaking of disulfide thus causing conformational changes [59].

\subsection{PL spectra of Au-CdSe hybrid NPs/QDs, and Au-BSA-CdSe, Au-Lysozyme-CdSe complex}

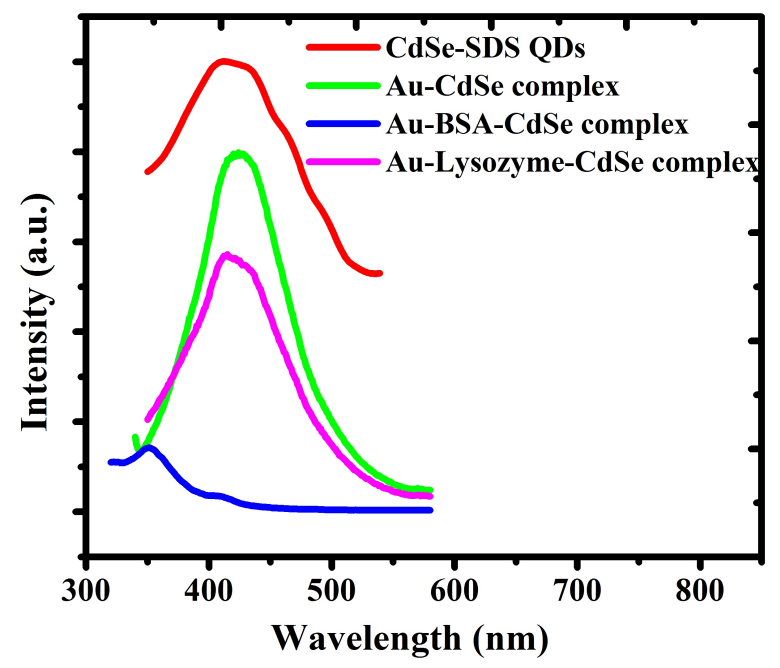

FIG. 5. PL spectra of CdSe-QDs, Au-CdSe NPs-QDs and their different protein conjugated hybrid systems

The PL spectra of Au NPs, CdSe QDs, Au-CdSe NPs-QDs and different protein conjugated systems are shown in Fig. 5 (excitation wavelengths, $\lambda_{e x}=300$ for all the samples). When Au NPs form a hybrid system with CdSeQDs, the emission maxima intensity of the QDs showed a quenching of intensity by $20 \%$. Similar behavior has been observed with the formation of Au-Lysozyme-CdSe NP- protein-QD system. In this case, the intensity has been reduced to $57 \%$ as compared to bare CdSe-SDS QDs. On the other hand, while replacing Lysozyme with BSA protein, 
a sharp decrease of PL intensity of the QDs (up to $\sim 15 \%$ ) has been observed. In addition, a significant blue shifting of the peak from $\sim 416 \mathrm{~nm}$ for the CdSe QDs to $\sim 344 \mathrm{~nm}$ has been observed in this system (Au-BSA-CdSe). In this case, the blue shifting of the emission peak in Au-BSA-CdSe hybrid system may signify a change in the tertiary structure of the BSA molecule with flexible conformation on the QDs surface by the SDS surfactant [60]. However, no blue shifting and hence, no effect of SDS surfactant on the modification of secondary has been observed for Lysozyme protein case. This is may be due to the highly stable behavior of the Lysozyme proteins [61].

While forming Au NPs-CdSe QDs system, a strong interaction between the plasmons of the metallic NPs and the excitons of the semiconductor QDs can be predicted to occur from the observed luminescence quenching of the QDs [57]. The conjugations of protein(s) with the Au NPs-CdSe QDs system(s) significantly influence the quenching phenomena by absorption of photons with their tryptophan moieties. The quenching of fluorescence intensity of the CdSe-QDs with conjugation of Au NPs and BSA or Lysozyme protein is due to the nonradiative energy transfer from QDs to metallic-semiconductor Au NPs and protein conjugated Au NPs [62].

The energy transfer efficiency can be measured experimentally by using the following equation [63]:

$$
E=1-\frac{F_{D A}}{F_{D}}
$$

where $F_{D A}$ is the integrated fluorescence intensity of the donor (CdSe-SDS QDs) in presence of the acceptor (Au NPs/ Au-BSA/ Au-Lysozyme NPs-protein complex) and $F_{D}$ is the integrated fluorescence intensity of the donor alone. In the present case, the energy transfer efficiency of the CdSe-SDS QDs to the Au NPs was 46\%. The corresponding value for the Lysozyme-conjugated Au NPs (Au-Lysozyme) was found to be 60\%, whereas, for the BSA conjugated $\mathrm{Au}$ NPs (Au-BSA), the transfer efficiency was $94 \%$.

In the classical Förster energy transfer mechanism, there should be significant overlapping between acceptor absorption spectra and donor emission spectra. In the present case(s), the overlapping can be possible by coupling of dipole of the QDs with the dipole of the LSPR field created by the Au NPs (or Au-protein complexes) [64]. As the LSPR is highly sensitive to the small changes in the surface environment, the coupling of CdSe QDs with the Au NPs and Au-BSA/Lysozyme significantly regulates the QDs fluorescent emission behavior.

(A)

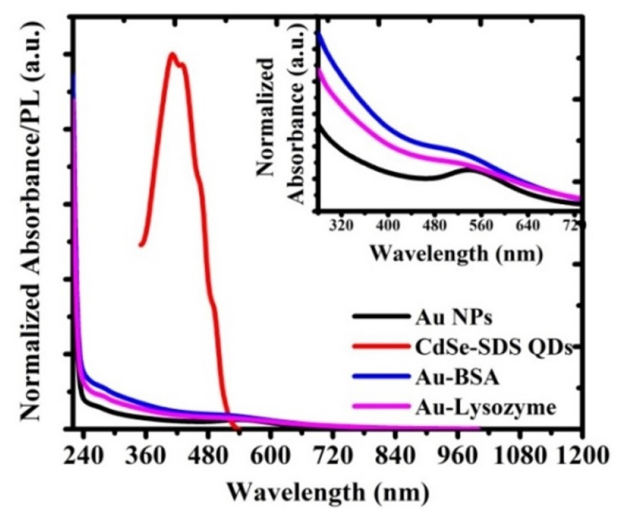

(B)

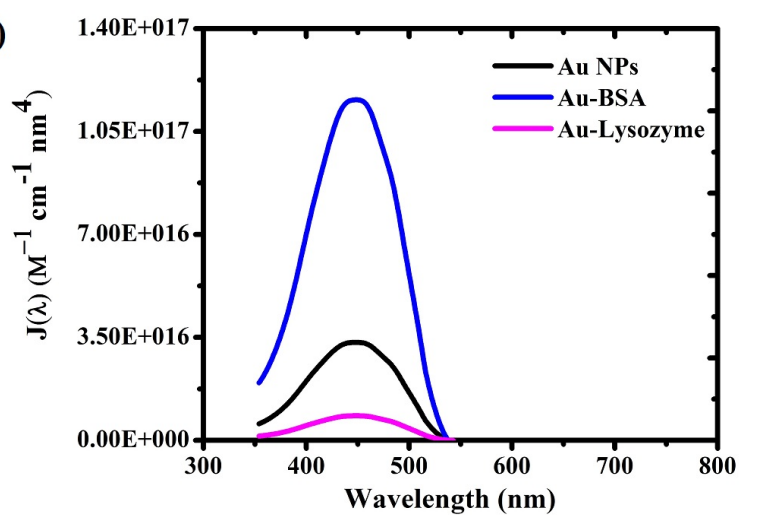

FIG. 6. (A) overlapping behavior (B) overlapping integral spectra between the normalized absorption spectra of Au NPs/ Au-BSA/ Au-Lysozyme complex and emission spectra of CdSe-SDS QDs

Fig. 6(A) shows the overlapping feature of the normalized absorption spectra of Au NPs/Au-BSA/Au-Lysozyme system and emission spectra of CdSe-SDS QDs. The corresponding overlapping integral spectra are shown in Fig. 6(B). The expression for overlapping integral is given by:

$$
J(\lambda)=\frac{\int_{0}^{\infty} F_{D}(\lambda) \varepsilon_{A}(\lambda) \lambda^{4} d \lambda}{\int_{0}^{\infty} F_{D}(\lambda) d \lambda}
$$

Here, $F_{D}(\lambda)$ is the integrated fluorescence intensity of the donor, $\varepsilon_{A}$ is the molar extinction coefficient of the acceptor, and $\lambda$ is the wavelength in nanometers. The required values of the $\varepsilon_{A}$ of the acceptors were calculated by using powerlaw expressions for the dependence of the maximum extinction coefficient on particle diameter [65]. Whereas, the diameter of acceptors, Au NPs, Au-BSA and Au-Lysozyme complex were obtained by using the Haiss equation [66].

The theoretically calculated values of size of the acceptors are depicted in the Table 2. The size of Au NPs $(\sim 1.63 \mathrm{~nm})$ is found to be comparable with the average size obtained from HRTEM $(\sim 2 \mathrm{~nm})$. The calculated values of $J(\lambda)$ are obtained as $3.45 \times 10^{16}, 8.5 \times 10^{15}, 1.19 \times 10^{17} \mathrm{M}^{-1} \mathrm{~cm}^{-1} \mathrm{~nm}^{4}$ for independent Au-CdSe, Au-BSA-CdSe 
TABLE 2. Parameters calculated for FRET

\begin{tabular}{|c|c|c|c|c|c|}
\hline Sample & $D_{(\text {theoretical })},(\mathrm{nm})$ & $\varepsilon_{A}$ & $J(\lambda)$ & $E$ & $d_{0}(\AA)$, FRET \\
\hline $\mathrm{Au}$ & 1.63 & $23.56 \times 10^{4}$ & $3.45 \times 10^{16}$ & 46 & 73.5 \\
\hline $\mathrm{Au}-$ Lysozyme & 1.07 & $5.87 \times 10^{4}$ & $8.5 \times 10^{15}$ & 60 & 58 \\
\hline $\mathrm{Au}-\mathrm{BSA}$ & 2.38 & $82 \times 10^{4}$ & $1.19 \times 10^{17}$ & 94 & 90 \\
\hline
\end{tabular}

and Au-Lysozyme-CdSe donor acceptor-pairs respectively. Thus, the overlap integral between the emission spectrum of the QD and the extinction spectrum of the Au NPs increases as the gold particle size increases. The overlapping integrals as well as the transfer efficiency are found to be highest in case of Au-BSA-CdSe FRET pair due to increased spectral overlap of the plasmonic band with the luminescence band of QDs. The coupling behavior of the QDs and the Au NPs (or Au-Protein) could be changed not only by the overlapping of the absorption spectra of Au NPs (or Au-Protein) and emission spectra of the QDs, it is also affected by the distance between absorber (Au, Au-BSA, Au-Lysozyme) and the emitter (CdSe QDs) [67].

To verify further the mechanism of energy transfer from the QDs to the Au NPs, we have examined the separation distance $\left(d_{0}\right)$ at which the energy transfer efficiency is $50 \%$

For FRET, $d_{0}$ can be expressed as:

$$
d_{0}=0.211\left(k^{2} J(\lambda) n^{-4} Q_{D}\right)^{1 / 6} .
$$

Here, $k^{2}$ is the dipole orientation factor and its value is $2 / 3$ for randomly oriented dipole, $\mathrm{n} \sim 1.33$ is the RI of the solvent, while $Q_{D}$ is the quantum yield of the donor. The calculated $d_{0}$ values for Au NPs, Au-BSA complex and Au-Lysozyme complexes are calculated to be 73.5, 90, $58 \AA$; respectively. Here, it is important to mention that for an ideal FRET pair, the typical Forster distance is in the range of 20-100 ̊̊ [67]. Hence, FRET can be likely to occur in all the systems.

\subsection{CdSe QDs, Au-CdSe Au-BSA-CdSe, Au-Lysozyme-CdSe systems as cancer cell probe}

Fig. 7A shows a series of imaging snap-shots of MDA-MB231 cancer cell treated with QDs and its different complex. The snapshots represent high biocompatibility and fluorescent behavior of the QDs and its complex in the cancer cellular environment.

Using image J-1.46r software, the quantitative analysis of the fluorescent counts can be predicted presuming complete localization of the QDs inside the cell. Fig. 7B highlights the areas of interest and selected background area of fluorescent images, marked with encircled regions. The corrected total counts of fluorescence (CTCF) intensity value can be calculated using the relation given by:

$$
\mathrm{CTCF}=\text { Integrated density }-(\text { Area } \times \text { mean fluorescent of background setting }) .
$$

Here, the integrated density (Int.Den.) of a fluorescent image is the sum of the values of the pixels in the selected regions. As shown in Fig. 7B(a-d), different selected fluorescent areas are labeled as A, B, C, D.... etc. and different selected background regions (region without fluorescence) are represented as bk1, bk2, bk3.........etc. Fig. 7C signifies a comparative view on representative histograms of the average CTCF and average Int.Den. obtained for different QDs system. These predict the biocompatibility and fluorescent behavior of the QDs with the treatment of cancer cell. Moreover, the fluorescence intensity indicates the level of the internalization of QDs by cells.

Note that, the fluorescence efficiency $(\mathrm{CTCF})$ of semiconductor $\mathrm{QDs}(\mathrm{Q})$ in the cancer cellular medium is dramatically quenched while conjugating (AQ) with metallic Au NPs (by factor of 31.4). Further reduction of the intensity of the QDs has been observed with formation of Au-Lysozyme-CdSe (AQL, by factor of 74) and Au-BSA-CdSe (AQB, by factor of 400), complex. The reduction of flourescence in the cancer cell environment is strongly resembled with the photoluminiscence quenching behavior as described above. Thus, the cellular uptake and bioimaging aspects are significantly affected by the metallic-semiconductor system and its bio complex. The significantly varied zeta potential values of the complex systems over the QDs (Table 1) is largely responsible for altering the cellular uptake processes $[68,69]$. Although the fluorescent intensity of the hybrid metal NPs-protein-QDs are found to be reduced as compared to a CdSe-SDS QD, the former will be more favorable than the latter ones. This is because of the higher cytotoxicity of the protein conjugated Au-CdSe systems against cancer cells relative to normal cells [70]. In presence of protein, there is elevation of reactive oxygen species (ROS) levels in cancer cells in the hybrid NPprotein-QDs [71-73]. At low levels, ROS may play an important role in molecular signaling, regulating fundamental biological processes such as cell viability, proliferation, migration, and differentiation. Increase of ROS levels may result in interference with basic cellular functions, leading to DNA damage and finally cell death. By using proteins in different concentrations and exposure times, the ROS elevation and hence the cancer cell death can precisely 
(A)

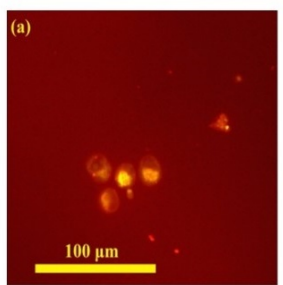

(B)
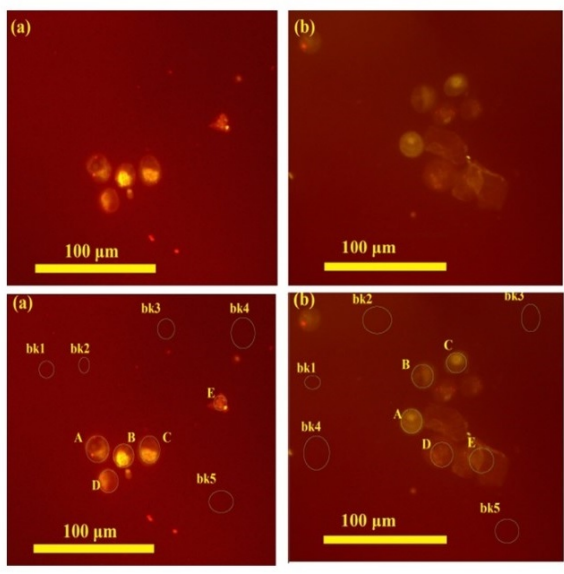

(C)

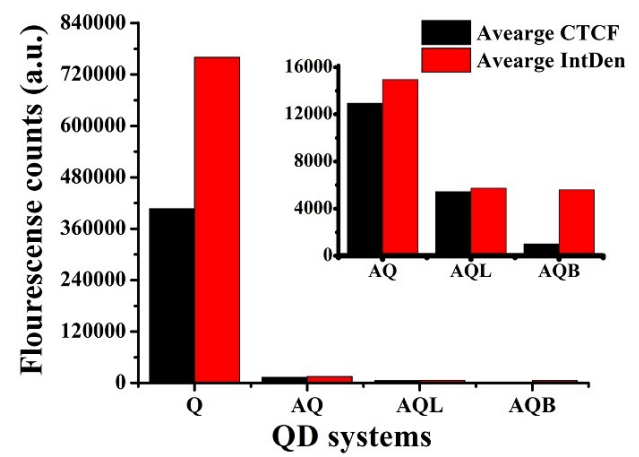

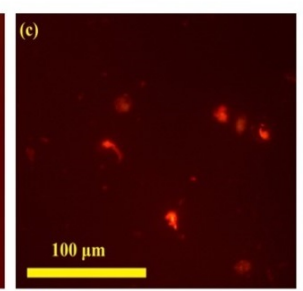
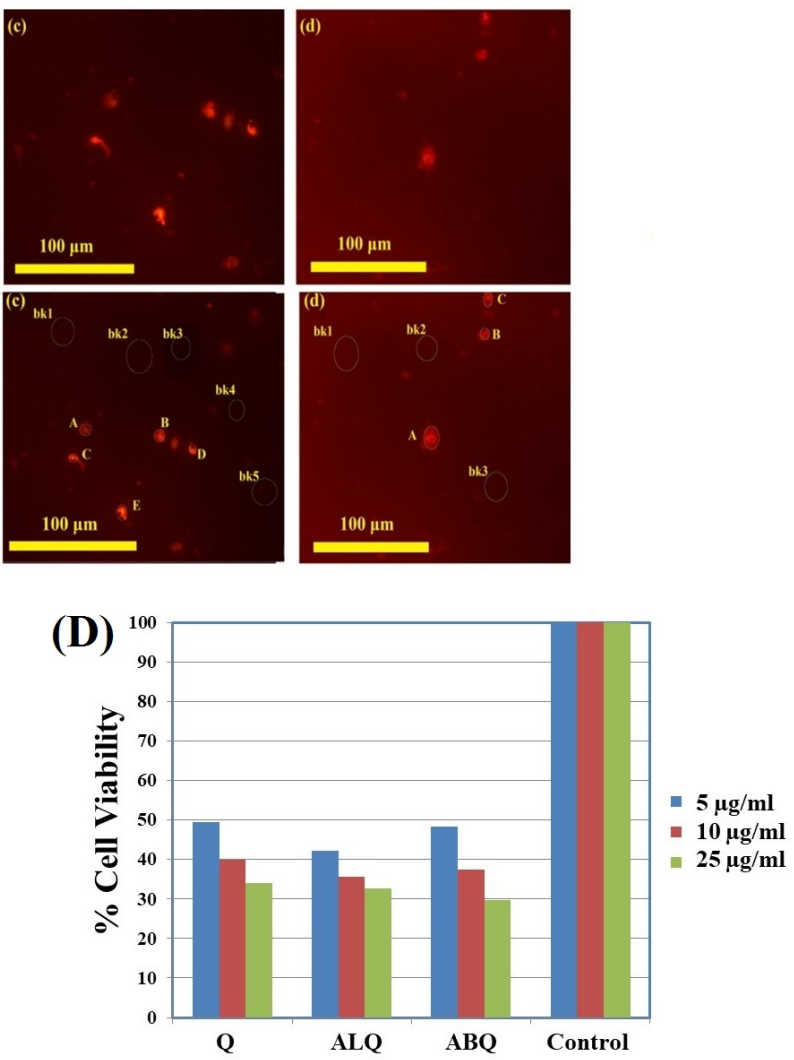

FIG. 7. Fluorescent imaging (using violate filter) of cancer cells with A(a) CdSe-SDS QDs (b) AuCdSe QDs (c) Au-Lysozyme-CdSe QDs(d) Au-BSA-CdSe QDs. The lower panel B shows selected fluorescent and background areas of fluorescent images respectively. Histograms representing the average value of Int.Den. and CTCF of different types QD systems is shown in C(D) cell viability study of cancer cellswith the treatment of CdSe-SDS QDs (sample Q), Au-BSA-CdSe (sample ABQ) and Au-Lysozyme-CdSe (ALQ) hybrid system

controlled [72]. While studying cell viability of the MDA-MB231 cancer cells (Figure 7D) with the treatment of CdSe-SDS QDs (sample Q), Au-BSA-CdSe (sample ABQ) and Au-Lysozyme-CdSe (ALQ) hybrid system, it was observed that cell viability decreases with the inclusion of BSA or Lysozyme proteins into protein unconjugated CdSe QDs. For $5 \mu \mathrm{g} / \mathrm{mL}$ QDs, the cell viability obtained as 50\%. Whereas, in the same concentration, the cell viabilities are decreased to $42 \%$ and $48 \%$ for Lysozyme and BSA conjugated Au-QDs samples; respectively. Similarly, with increase of concentration up to $10 \mu \mathrm{g} / \mathrm{mL}$ of Au-Lysozyme-QDs and Au-BSA-QDs, the cell viability decreased by the factor of $\sim 1.12$ and $\sim 1.07$ as compared to protein unconjugated QDs. These factors are $\sim 1.04$ and 1.14; respectively for the concentration of $25 \mu \mathrm{g} / \mathrm{mL}$. Thus, it signifies the higher efficiency of cancer cell death by inclusion proteins in the Au-CdSe system.

\section{Conclusions}

A simple colloidal route has been followed for the synthesis of Au NPs, CdSe QDs and their hybrid system (Au$\mathrm{CdSe}$ ). A special emphasis was given to the conjugation of BSA and Lysozyme proteins to this metal-semiconductor complex. HR-TEM data revealed the enhancement of average diameter of Au NPs while conjugating with CdSe QDs from $2 \mathrm{~nm}$ to $\sim 3.8 \mathrm{~nm}$. Moreover, considering different sections and their corresponding FFT and IPF of HRTEM image of Au-CdSe hybrid, the presence of CdSe QDs as outer shell of Au NPs can be predicted. The zeta potential measurement signifies the enhancement of stability of Au NPs while forming Au-CdSe, Au-BSA-CdSeandAuLysozyme-CdSe hybrid system. Multipolar excitations and radiative damping along with strong interaction between plasmons of Au NPs and excitons of CdSe QDs in hybrid Au-CdSe system may cause the appearance of red shifted 
and broadened absorption peaks at $\sim 545 \mathrm{~nm}$ and $\sim 303 \mathrm{~nm}$ as compared to parent Au and CdSe QDs. Photoluminescence study shows significant reduction of peak maxima intensity of CdSe QDs while conjugating with Au NPs and Au-BSA or Au-Lysozyme complex. There is expected to occur high probability of FRET phenomenon in between the donor CdSE-SDS QDs and acceptor(s) Au-PVP NPs (or Au-BSA, Au-Lysozyme). The energy transfer efficiency from acceptor to donor was found to be as high as 94\% for the FRET pair CdSe and Au-BSA. From the fluorescence microscopy imaging data, it can be observed that, the synthesized QDs and its complex with Au NPs and Au-BSA/Lysozyme are highly biocompatible with a high fluorescent contrast in cancer cell environment. The significantly varied zeta potential values of the complex systems over the QDs influenced the cellular uptake processes.

\section{Acknowledgement}

The author R Sarma thanks DBT-New Delhi for financial support under DBT-RA scheme, Material Science Division, CSIR-NEIST, Jorhat for providing me nanoparticle synthetic lab facility and UV-Vis, Zeta potential measurement. The authors gratefully acknowledge Analytical Chemistry Group, CSIR-NEIST for FTIR data, SAIF, NEHU, Shillong for HR-TEM measurements, Department of Physics-IIT Guwahati for PL study and Dr. Manash Jyoti Kashyap for providing important inputs in restructuring and editing the manuscript extensively.

\section{Conflict of interest}

On behalf of all authors, the corresponding author states that there is no conflict of interest.

\section{References}

[1] Wild C.P. Cancer control: a reminder of the need for a balanced approach between prevention and treatment, 2014.

[2] Yang C., et al. Gold nanoparticle mediated combined cancer therapy. Cancer Nanotechnology, 2018, 9(1), P. 4.

[3] Hartshorn C.M., et al. Nanotechnology Strategies To Advance Outcomes in Clinical Cancer Care. ACS Nano, 2018, 12(1), P. 24-43.

[4] Grodzinski P., et al. Integrating Nanotechnology into Cancer Care. ACS Nano, 2019, 13(7), P. 7370-7376.

[5] Vines J.B., et al. Gold Nanoparticles for Photothermal Cancer Therapy. Frontiers in Chemistry, 2019, 7(167).

[6] Singh P., et al. Gold Nanoparticles in Diagnostics and Therapeutics for Human Cancer. International journal of molecular sciences, 2018, 19(7), P. 1979.

[7] Riley R.S., Day E.S. Gold nanoparticle-mediated photothermal therapy: applications and opportunities for multimodal cancer treatment. Wiley interdisciplinary reviews. Nanomedicine and nanobiotechnology, 2017, 9(4), P. 10.

[8] Xiao W., et al. Normalizing Tumor Vessels To Increase the Enzyme-Induced Retention and Targeting of Gold Nanoparticle for Breast Cancer Imaging and Treatment. Molecular Pharmaceutics, 2017, 14(10), P. 34890-3498.

[9] Lara-Cruz C., et al. Gold nanoparticle uptake is enhanced by estradiol in MCF-7 breast cancer cells. International journal of nanomedicine, 2019, 14, P. 2705-2718.

[10] Hoshyar R., et al. A novel green one-step synthesis of gold nanoparticles using crocin and their anti-cancer activities. Journal of Photochemistry and Photobiology B: Biology, 2016, 159, P. 237-242.

[11] Connor E.E., et al. Gold nanoparticles are taken up by human cells but do not cause acute cytotoxicity. Small, $2005,1(3)$, P. $325-327$.

[12] Dimitri A., et al. Semiconductor nanostructures in biological applications. Journal of Physics: Condensed Matter, $2005,17(26)$, P. R637.

[13] Sarma R., et al. Physical and biophysical assessment of highly fluorescent, magnetic quantum dots of a wurtzite-phase manganese selenide system. Nanotechnology, 2014, 25(27), P. 275101.

[14] Chu M., et al. The therapeutic efficacy of CdTe and CdSe quantum dots for photothermal cancer therapy. Biomaterials, 2012, 33(29), P. 70717083.

[15] Zayed J., Philippe S. Acute oral and inhalation toxicities in rats with cadmium telluride. International journal of toxicology, 2009, 28(4), P. 259-265.

[16] Hardman R. A toxicologic review of quantum dots: toxicity depends on physicochemical and environmental factors. Environmental health perspectives, 2006, 114(2), P. 165-172.

[17] Sarma R., Mohanta D. Luminescence and bio-imaging response of thio-glycolic acid (TGA) and sodium dodecyl sulfate (SDS)-coated fluorescent cadmium selenide quantum dots. Journal of Luminescence, 2015, 161, P. 395-402.

[18] Chen F., Gerion D. Fluorescent CdSe/ZnS Nanocrystal-Peptide Conjugates for Long-term, Nontoxic Imaging and Nuclear Targeting in Living Cells. Nano Letters, 2004, 4(10), P. 1827-1832.

[19] Guo G., et al. Probing the cytotoxicity of CdSe quantum dots with surface modification. Materials Letters, 2007, 61(8), P. 1641-1644.

[20] Achermann M. Exciton-Plasmon Interactions in Metal-Semiconductor Nanostructures. The Journal of Physical Chemistry Letters, 2010, 1(19), P. 2837-2843.

[21] Feng A.L., et al. Distance-dependent plasmon-enhanced fluorescence of upconversion nanoparticles using polyelectrolyte multilayers as tunable spacers. Scientific reports, 2015, 5 .

[22] Govorov A.O., Lee J., and Kotov N.A. Theory of plasmon-enhanced Förster energy transfer in optically excited semiconductor and metal nanoparticles. Physical Review B, 2007, 76(12), P. 125308.

[23] Fedutik Y., et al. Exciton-plasmon-photon conversion in plasmonic nanostructures. Physical review letters, $2007,99(13)$, P. 136802.

[24] Medintz I.L., et al. Proteolytic activity monitored by fluorescence resonance energy transfer through quantum-dot-peptide conjugates. Nature materials, 2006, 5(7), P. 581-589.

[25] Fuller M.A. and Köper I. Biomedical applications of polyelectrolyte coated spherical gold nanoparticles. Nano Convergence, 2019,6 (1), P. 11.

[26] Hezinger A., Teßmar J., and Göpferich A. Polymer coating of quantum dots-a powerful tool toward diagnostics and sensorics. European Journal of Pharmaceutics and Biopharmaceutics, 2008, 68(1), P. 138-152. 
[27] Hamouda R.A., Abd El-Mongy M., and Eid K.F. Comparative study between two red algae for biosynthesis silver nanoparticles capping by SDS: Insights of characterization and antibacterial activity. Microbial pathogenesis, 2019, 129, P. $224-232$.

[28] Rajh T., Micic O.I., and Nozik A.J. Synthesis and characterization of surface-modified colloidal cadmium telluride quantum dots. The Journal of Physical Chemistry, 1993, 97(46), P. 11999-12003.

[29] Zhang Y. and Clapp A. Overview of stabilizing ligands for biocompatible quantum dot nanocrystals. Sensors, 2011, 11(12), P. 11036-11055.

[30] Gerion D., et al. Synthesis and properties of biocompatible water-soluble silica-coated CdSe/ZnS semiconductor quantum dots. The Journal of Physical Chemistry B, 2001, 105(37), P. 8861-8871.

[31] Wang P., et al. Interaction of gold nanoparticles with proteins and cells. Science and technology of advanced materials, 2015, 16(3), P. 034610034610.

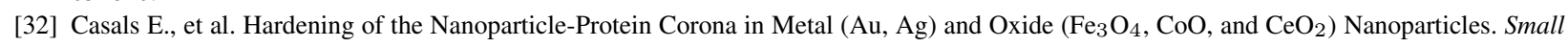
(Weinheim an der Bergstrasse, Germany), 2011, 7, P. 3479-86.

[33] Malek M., et al. Charged and Neutral Au Nanoparticles Interact Differently with Langmuir Film-Based Synthetic Membranes: Implications for Nanoparticle Uptake and Membrane Protein Activity. ACS Applied Nano Materials, 2020, 3(9), P. 9276-9284.

[34] Clapp A.R., Goldman E.R., and Mattoussi H. Capping of CdSe-ZnS quantum dots with DHLA and subsequent conjugation with proteins. Nature Protocols, 2006, 1(3), P. 1258-1266.

[35] Foubert A., et al. Bioconjugation of quantum dots: Review and impact on future application. TrAC Trends in Analytical Chemistry, 2016, 83, P. $31-48$.

[36] Ding S., et al. Protein-based nanomaterials and nanosystems for biomedical applications: A review. Materials Today, 2021,43 , P. 166-184.

[37] Spicer C.D., et al. Peptide and protein nanoparticle conjugates: versatile platforms for biomedical applications. Chemical Society reviews, 2018, 47(10), P. 3574-3620.

[38] Xie J., Lee J.Y., and Wang D.I.C. Synthesis of Single-Crystalline Gold Nanoplates in Aqueous Solutions through Biomineralization by Serum Albumin Protein. The Journal of Physical Chemistry C, 2007, 111(28), P. 10226-10232.

[39] Sarkar S., et al. Protein nanocomposites: Special inferences to Lysozyme based nanomaterials. International Journal of Biological Macromolecules, 2020, 151, P. 467-482.

[40] Das T., et al. Functionalisation of Polyvinylpyrrolidone on Gold Nanoparticles Enhances Its Anti-Amyloidogenic Propensity towards Hen Egg White Lysozyme. Biomedicines, 2017, 5(2), P. 19.

[41] Damjanovic V. and Thomas D. The use of polyvinylpyrrolidone as a cryoprotectant in the freezing of human lymphocytes. Cryobiology, 1974, 11(4), P. 312-316.

[42] Prasad M., Palepu R., and Moulik S.P. Interaction between sodium dodecyl sulfate (SDS) and polyvinylpyrrolidone (PVP) investigated with forward and reverse component addition protocols employing tensiometric, conductometric, microcalorimetric, electrokinetic, and DLS techniques. Colloid and Polymer Science, 2006, 284(8), P. 871-878.

[43] Romani A., Gehlen M., and Itri R. Surfactant-Polymer Aggregates Formed by Sodium Dodecyl Sulfate, Poly( N -vinyl-2-pyrrolidone), and Poly(ethylene glycol). Langmuir: the ACS journal of surfaces and colloids, 2005, 21, P. 127-33.

[44] Kavlak S. and Guner A. Intermolecular interactions between bovine serum albumin and certain water-soluble polymers at various temperatures. Journal of applied polymer science, 2006, 100(2), P. 1554-1560.

[45] Jette E.R. and Foote F. Precision determination of lattice constants. The Journal of Chemical Physics, 1935, 3(10), P. 605-616.

[46] Freeman D., Mair S., and Barnea Z. The structure and Bijvoet ratios of cadmium selenide. Acta Crystallographica Section A: Crystal Physics, Diffraction, Theoretical and General Crystallography, 1977, 33(3), P. 355-359.

[47] Li Y. and Chopra N. Gold nanoparticle integrated with nanostructured carbon and quantum dots: synthesis and optical properties. Gold Bulletin, 2015, 48(1), P. 73-83.

[48] Paolino D., et al. Drug delivery systems. Encyclopedia of medical devices and instrumentation, 2006.

[49] Joseph E. and Singhvi G., Chapter 4 - Multifunctional nanocrystals for cancer therapy: a potential nanocarrier, in Nanomaterials for Drug Delivery and Therapy, A.M. Grumezescu, Editor. 2019, William Andrew Publishing, P. 91-116.

[50] Honary S. and Zahir F. Effect of Zeta Potential on the Properties of Nano-Drug Delivery Systems - A Review (Part 1). Tropical Journal of Pharmaceutical Research, 2013, 12.

[51] Behera M. and Ram S. Inquiring the mechanism of formation, encapsulation, and stabilization of gold nanoparticles by poly(vinyl pyrrolidone) molecules in 1-butanol. Applied Nanoscience, 2013, 4.

[52] Borodko Y., et al. Probing the interaction of poly (vinylpyrrolidone) with platinum nanocrystals by UV-Raman and FTIR. The Journal of Physical Chemistry B, 2006, 110(46), P. 23052-23059.

[53] Behera M. and Ram S. Inquiring the mechanism of formation, encapsulation, and stabilization of gold nanoparticles by poly (vinyl pyrrolidone) molecules in 1-butanol. Applied Nanoscience, 2014, 4(2), P. 247-254.

[54] Viana R.B., da Silva A.B.F., and Pimentel A.S. Infrared Spectroscopy of Anionic, Cationic, and Zwitterionic Surfactants. Advances in Physical Chemistry, 2012, 2012, P. 14.

[55] Chaudhary A., et al. Morphological effect of gold nanoparticles on the adsorption of bovine serum albumin. Physical Chemistry Chemical Physics, 2014, 16(38), P. 20471-20482.

[56] Kvítek O., et al. Noble metal nanostructures influence of structure and environment on their optical properties. Journal of Nanomaterials, 2013, 2013, P. 111.

[57] Haldar K.K., et al. Photophysical properties of Au-CdTe hybrid nanostructures of varying sizes and shapes. ChemPhysChem, 2012, 13(17), P. 3989-3996.

[58] Templeton A.C., et al. Solvent refractive index and core charge influences on the surface plasmon absorbance of alkanethiolate monolayerprotected gold clusters. The Journal of Physical Chemistry B, 2000, 104(3), P. 564-570.

[59] Singh S., et al. Conjugation of nano and quantum materials with bovine serum albumin (BSA) to study their biological potential. Journal of Luminescence, 2013, 141, P. 53-59.

[60] Vaishanav S.K., et al. Adsorption Kinetics and Binding Studies of Protein Quantum Dots Interaction: A Spectroscopic Approach. Journal of Fluorescence, 2016, 26(3), P. 855-865.

[61] Blout E.R. and L. Visser, Elastase. II. Optical properties and the effects of sodium dodecyl sulfate. Biochemistry, 1971, 10(5), P. 743-752. 
[62] Xia Y., Song L., and Zhu C. Turn-On and Near-Infrared Fluorescent Sensing for 2,4,6-Trinitrotoluene Based on Hybrid (Gold Nanorod)(Quantum Dots) Assembly. Analytical Chemistry, 2011, 83(4), P. 1401-1407.

[63] Clapp A.R., et al. Fluorescence resonance energy transfer between quantum dot donors and dye-labeled protein acceptors. Journal of the American Chemical Society, 2004, 126(1), P. 301-310.

[64] Li M., et al. Size-Dependent Energy Transfer between CdSe/ZnS Quantum Dots and Gold Nanoparticles. The Journal of Physical Chemistry Letters, 2011, 2(17), P. 2125-2129.

[65] Navarro J.R. and Werts M.H. Resonant light scattering spectroscopy of gold, silver and gold-silver alloy nanoparticles and optical detection in microfluidic channels. Analyst, 2013, 138(2), P. 583-592.

[66] Haiss W., et al. Determination of size and concentration of gold nanoparticles from UV-vis spectra. Analytical chemistry, 2007, 79(11), P. 4215-4221.

[67] Li X., et al. Plasmon-Emitter Hybrid Nanostructures of Gold Nanorod-Quantum Dots with Regulated Energy Transfer as a Universal NanoSensor for One-step Biomarker Detection. Nanomaterials (Basel, Switzerland), 2020, 10(3), P. 444.

[68] Patil S., et al. Protein adsorption and cellular uptake of cerium oxide nanoparticles as a function of zeta potential. Biomaterials, 2007, 28(31), P. 4600-4607.

[69] Zhao M.-X. and Zeng E.-Z. Application of functional quantum dot nanoparticles as fluorescence probes in cell labeling and tumor diagnostic imaging. Nanoscale Research Letters, 2015, 10(1), P. 1-9.

[70] Azizi M., et al. Anti-cancerous effect of albumin coated silver nanoparticles on MDA-MB 231 human breast cancer cell line. Scientific Reports, 2017, 7(1), P. 5178.

[71] Matulionyte M., et al. Photoluminescent Gold Nanoclusters in Cancer Cells: Cellular Uptake, Toxicity, and Generation of Reactive Oxygen Species. International journal of molecular sciences, 2017, 18(2), P. 378.

[72] Dong L., et al. Cytotoxicity of BSA-Stabilized Gold Nanoclusters: In Vitro and In Vivo Study. Small, $2015,11$.

[73] Poderys V., et al. Protein-stabilized gold nanoclusters for PDT: ROS and singlet oxygen generation. Journal of Photochemistry and Photobiology B: Biology, 2020, 204, P. 111802. 